\title{
Watney Solid +/- Void
}

\section{KORY BIEG}

The University of Texas at Austin

Watney Solid +/- Void is an art installation for SXSW, the premier music, film, and technology festival in Austin, TX. The design process began with a catalogue of nearly 150 forms. After several iterations and further development of three schemes, a single form was selected.

Watney Solid +/- Void consists of three main components: a steel plate base, 21 individual fragments, and the outer skin. The fragments were developed through parametrically based computation methods and real-time structural analysis. Multiple iterations were tested for their ability to withstand dead and live loads. Each individual fragment is comprised of three parts: the waffle, the end caps, and the offset end-caps. The end-caps are secured to the waffle through teeth that hinge from the edge of each individual waffle element. The 2 " offset end caps are then mechanically attached to the outer surface, causing a slight reveal at the seam between fragments, accentuating the fractured nature of the form.

Watney Solid +/- Void provides a new take on a conventional structure system - the waffle rib system. By treating each part as an independent self-structuring, uniquely formal object, Watney Solid +/- Void disentangles the parts from a top-down structural scheme. Consequently, the assembly of multi-directional waffles contained by the surface of each part, once assembled, produces a whole that is much stronger than had a conventional rib system been used. Furthermore, the manifestation of each part's particular structural solution is, like the object itself, guided by an internal logic that relates to other properties of the part in which it is contained, as well as conditions imposed by the whole and contextual cues (including occupation, views and environmental/cultural responsiveness).

PROJECT TEAM: Maxwell Baird, Bruno Canales, Mandy Chung, Annabel Cook, Jayme Greene, Christina Hausle, James Holliday, Maxine Kraft, Renica Navea, Robert Orth, Kelsey Osborne, Andrew Tamez, Raquel Valdez Arias 

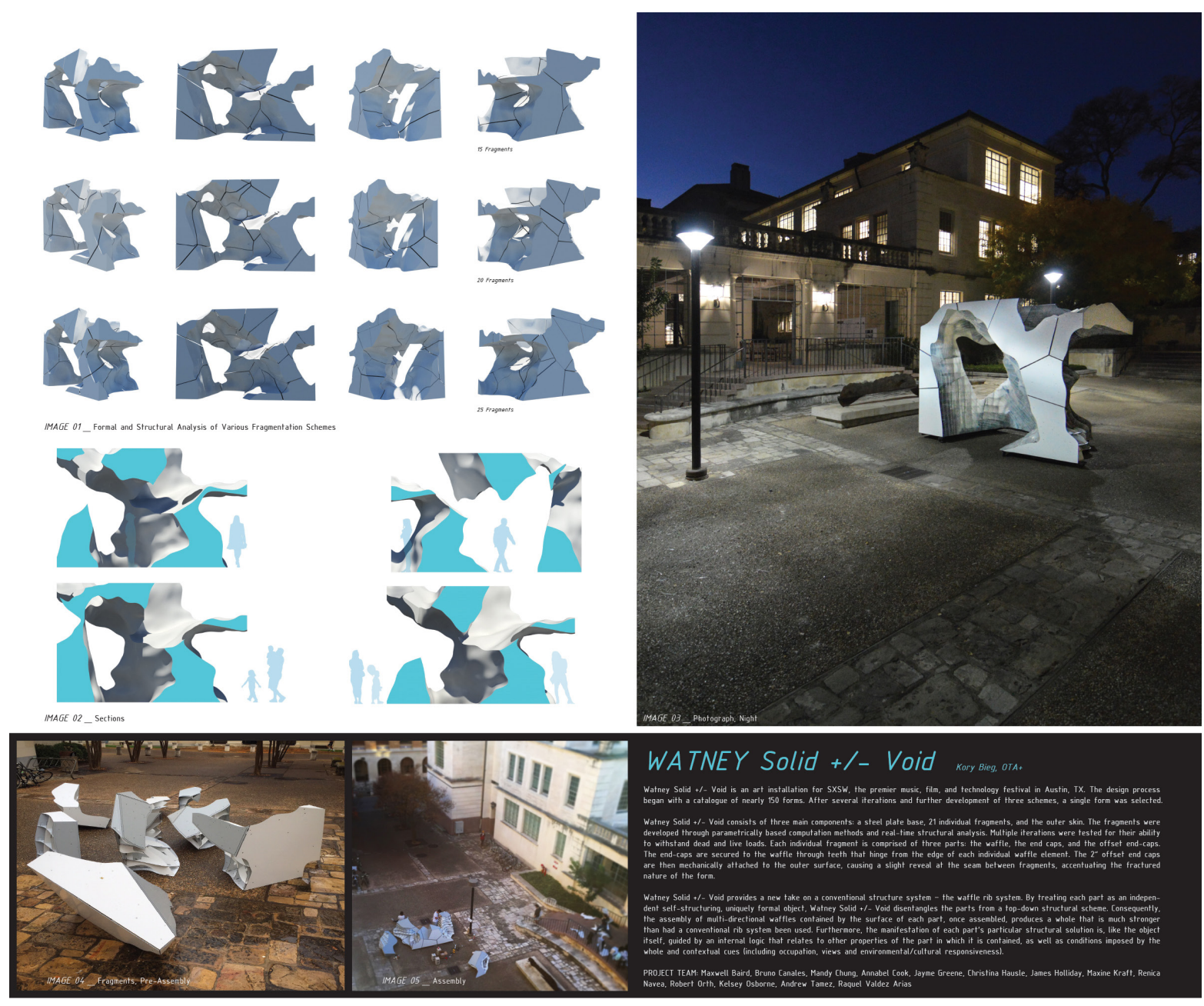

WATNEY Solid +/- Void kary beaco ora

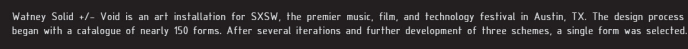

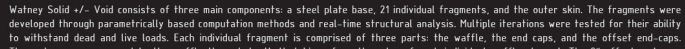

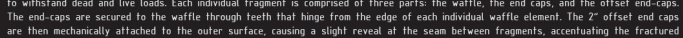

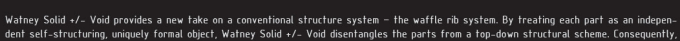

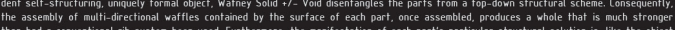

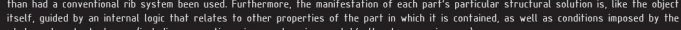

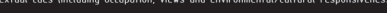

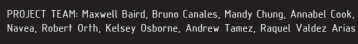
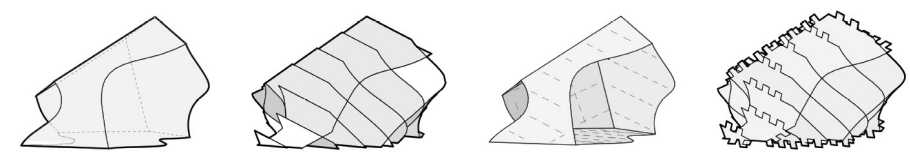

$B$ B

James Holliday, Maxine Kraft. Renica
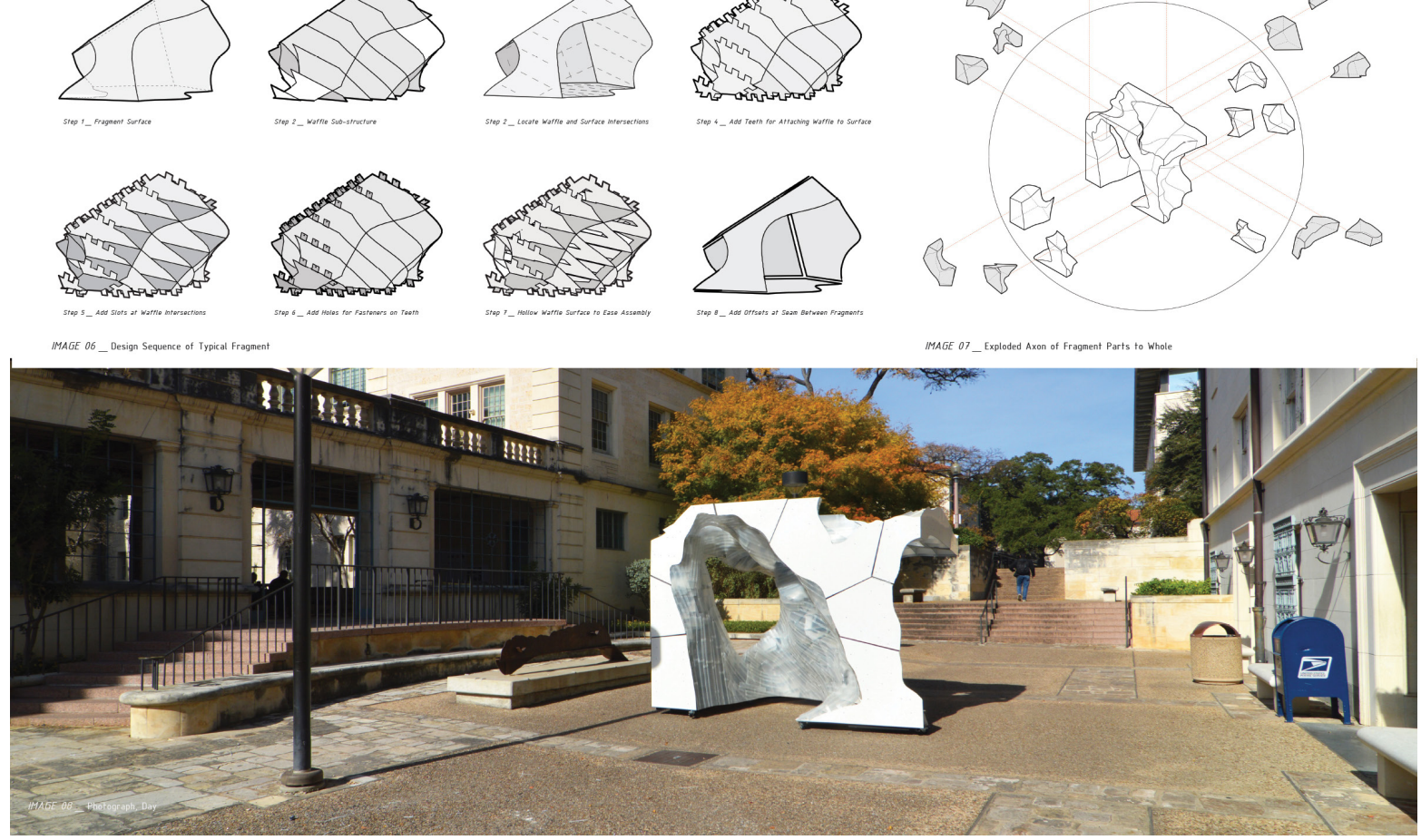